Revue d'histoire de l'Amérique française

ZAS REVUE D.HISTOIRE DE L'AMÉRIQUE FRANÇAISE

\title{
L’altérité dans l'imaginaire occidental : fonction manifeste, fonction occulte
}

\section{$\dagger$ Thierry Hentsch}

Volume 59, numéro 3, hiver 2006

URI : https://id.erudit.org/iderudit/013084ar

DOI : https://doi.org/10.7202/013084ar

Aller au sommaire du numéro

Éditeur(s)

Institut d'histoire de l'Amérique française

ISSN

0035-2357 (imprimé)

1492-1383 (numérique)

Découvrir la revue

Citer cet article

Hentsch, †. (2006). L'altérité dans l'imaginaire occidental : fonction manifeste, fonction occulte. Revue d'histoire de l'Amérique française, 59(3), 347-356.

https://doi.org/10.7202/013084ar d'utilisation que vous pouvez consulter en ligne.

https://apropos.erudit.org/fr/usagers/politique-dutilisation/ 


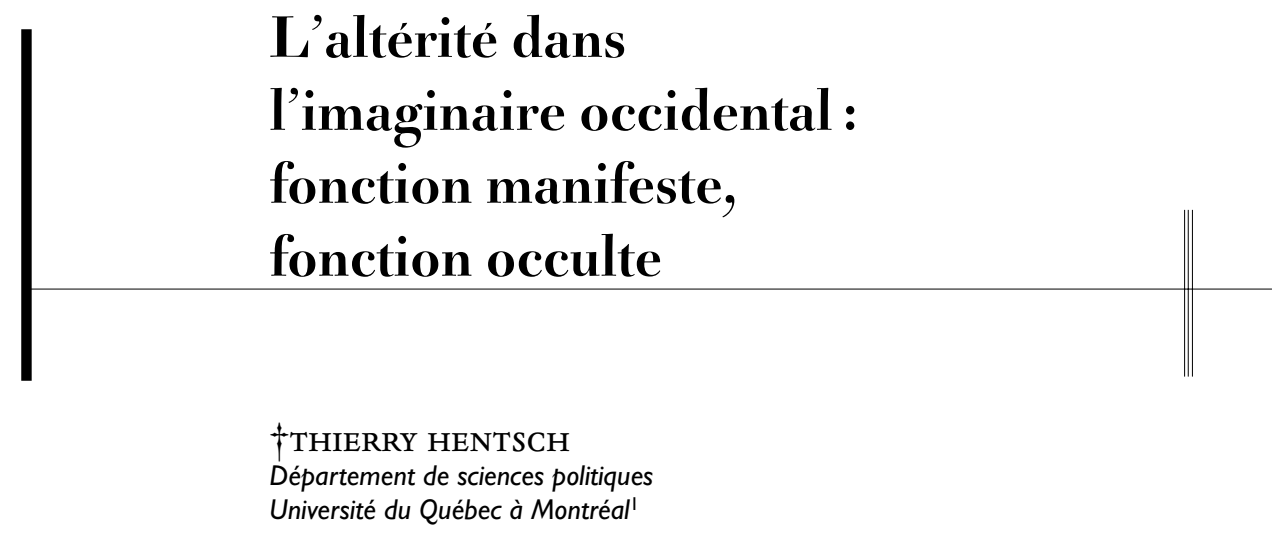

\section{MIROIRS}

L'Autre dans le miroir de l'histoire, la proposition, pour alléchante qu'elle soit, présente d'emblée un double paradoxe: l'Autre est indissociable du même, et l'histoire partiellement indistincte de la mythologie. Il ne s'agit pas d'allumer une mauvaise dispute sur mythologie et histoire ni de nier la possibilité de la rigueur historique. Placer côte à côte mythe et histoire, ici, c'est évoquer l'indistinction de la ligne qui les sépare. Or cette indistinction se retrouve à la frontière du même et de l'Autre. C'est dans le rapport de l'identité à l'altérité que la dimension mythique de l'histoire apparaît la plus prégnante. La question identitaire et la question des origines, pour toute société, baignent dans le même imaginaire. Voilà ce qui fait de l'histoire un miroir. Mais la réflexion est réversible : l'histoire à son tour s'inscrit dans le miroir de l'Autre. C'est ce double mouvement réflexif qu'il s'agit donc de suivre ici à travers l'exemple des rapports - plus tendus que jamais - entre l'Islam et l'Occident. Exercice de rhétorique qui n'est pas dépourvu d'importance, dans un monde où la parole, de part et d'autre, est une arme aussi redoutable que les bombes larguées du ciel ou

1. Notre collègue Thierry Hentsch étant décédé sans avoir pu revoir son texte, une révision minimale a été faite par Jean-Marie Fecteau. Il s'est essentiellement agi de retouches tenant au passage de la forme orale à la forme écrite et de l'ajout de quelques références en note. 
posées dans les gares. Paroles et massacres agissent en étroite liaison dans le train actuel des choses. Et au train où elles ne cessent d'aller, justement, les choses risquent fort de se gâter au-delà de toute mesure. L'histoire nous rattrape brutalement, nous, le monde, nous musulmans, juifs, chrétiens ou non croyants. Et l'Autre est le mur vers lequel chacun fonce les yeux fermés, en toute bonne conscience, convaincu qu'il ne fait que se défendre et accomplir son devoir.

\section{VÉRITÉ}

$S$ 'il n'est ici question que des trois principales religions monothéistes qui ont la Méditerranée orientale comme bassin, c'est qu'elles ont une histoire commune et une attitude comparable envers la question de la vérité, encore que, de ce point de vue, le judaïsme diffère sensiblement de ses deux rejetons chrétien et musulman. À cette question de la vérité, les religions ou systèmes de croyance d'Asie de l'Est ne prennent aucune part, et, soit dit en passant, cette distance apparaît comme une chance pour le monde. Si on englobe les non-croyants, c'est que la laïcité (pour lui donner cette appellation insatisfaisante), ce quatrième larron de la foire d'empoigne que nous vivons, est impensable en dehors de l'évolution même du monothéisme (il est douteux que la question de la laïcité se pose pour un Chinois, pour un Japonais et, à supposer qu'elle ait quelque résonance en Inde, c'est probablement en tant que reliquat de la présence coloniale et en raison de l'actuelle cohabitation entre islam et hindouisme dans le sous-continent). La laïcité est d'abord et avant tout le produit du christianisme et, plus encore, de la chrétienté occidentale. Le terme «Occident», aussi imprécis et abusif soit-il, a l'avantage d'en rendre implicitement compte en enfermant le christianisme latin et l'Occident moderne dans la même constellation historique. Les temps modernes sont toujours plus chrétiens que les modernes d'hier et d'aujourd'hui ne l'imaginent ou ne l'acceptent, tout comme l'athéisme est bien plus tributaire du monothéisme qu'il ne le voudrait.

\section{CONTINUITÉ}

La continuité, qui de la foi chrétienne conduit au credo de la modernité, a une grande incidence sur notre rapport à l'Autre, précisément de ce que nous avons les yeux rivés sur la rupture. C'est de croire que l'Occident moderne a rompu avec l'emprise de la religion et du religieux sur nos sociétés que vient en partie l'ambiguilté qui marque notre rapport aux sociétés musulmanes et notre regard sur l'Islam. Ces sociétés, à nos yeux, 
n'ont «pas encore» accompli le pas qui les affranchirait de l'emprise que la religion exerce abusivement sur elles, et dans la mesure où elles résistent devant la «nécessité» de cet affranchissement elles vont à contre-courant de l'histoire.

Ainsi va l'argument. Ses prémisses demandent à être questionnées. Le questionnement est complexe et semé d'embûches. Il met en jeu des facteurs psychiques et historiques étroitement imbriqués, que l'analyse a d'autant plus de peine à démêler que l'analyste est lui-même pris dans cet écheveau. L'auteur de ces lignes devrait exposer ici les motivations plus ou moins conscientes qui l'attachent depuis tant d'années à ces questions. Je prie simplement le lecteur de croire que c'est un aspect des choses, cette équation personnelle, auquel il essaie de rester aussi attentif que possible, espérant que cette attention sera perceptible dans les propos qui suivent.

\section{PSYCHISME}

Par une sorte d'ironie de l'histoire, les fondements psychiques du malentendu fondamental qui régit notre rapport à l'Autre sont inscrits dans les Évangiles. C'est la fameuse parabole de la paille et de la poutre. Dans le dépistage de la paille que nous cherchons dans l'œil de l'Autre, nous sommes aveuglés par la poutre qui obscurcit le nôtre. On pourrait s'en tenir à cette parabole à condition d'en explorer toutes les implications. Ses interprétations pêchent souvent par leur superficialité : nous sommes plus attentifs aux imperfections d'autrui qu'à nos propres défauts. Mais qu'est-ce qu'avoir une poutre dans l'œil qu'on ne voit pas, qu'on ne sent pas. Le plus intrigant dans cette métaphore est que la poutre ne nous empêche pas de voir, et l'idée qu'elle nous aveugle, à proprement parler, ne tient pas. Il y a donc là une énormité invisible qui nous empêche non pas tant de voir que de comprendre ce qui se passe dans le regard que nous portons sur l'Autre. Ce à quoi nous restons métaphoriquement aveugle est notre propre regard. À proprement parler, d'ailleurs, ce regard, le nôtre, est ce qu'il y a pour nous de plus radicalement irregardable. C'est ce que nous ne verrons jamais de nous. Car le regard que nous observons dans le miroir ne peut que se regarder soi-même dans l'acte de se regarder, sans du tout surprendre notre regard sur le monde. Le miroir est un trompe-l'œil. Ce n'est donc que dans le regard de l'Autre que nous pouvons déchiffrer quelque chose de notre regard sur le monde. L’Autre devient le miroir de notre regard sur les choses mais, à supposer que nous ayons conscience que l'Autre nous renvoie quelque chose de notre propre 
regard, ce miroir-là se révèle à son tour trompeur de ce que nous ne comprenons pas bien ce qu'il réfléchit. L’Autre est le plus souvent éprouvé comme injuste dans le regard qu'il porte sur nous, notamment - et ce n'est pas le moindre paradoxe - lorsqu'il se met à critiquer le regard négateur, objectal, que nous posons sur lui.

Nous croyons être "objectif» de ce que nous posons l'Autre comme objet - objet scientifique d'étude, notamment. Mais, depuis le temps, la lecture de Hegel devrait au moins nous amener à reconnaître que cette objectivation n'est qu'un moment partiel et insuffisant de notre regard (si sophistiqué et savant soit-il) ${ }^{2}$. L'Autre est un sujet qui parcourt vers nous le même chemin. Toute conscience consciente d'elle-même est nécessairement amenée à reconnaître cette réciprocité qui, une fois reconnue, exige un autre niveau de conscience. Il ne s'agit nullement ici de dialogue, mais du parcours que fait pour soi toute conscience qui fait entrer l'altérité comme une conscience égale à la sienne dans le circuit qu'elle accomplit pour regarder le monde et se situer dans le monde.

Hegel trace ce cheminement idéal. Freud en signale les difficultés, notamment dans son célèbre texte sur Das Unheimliche ${ }^{3}$, ordinairement (et malheureusement) traduit par "L’inquiétante étrangeté». Das Unheimliche est au sens propre l'in-quiet, le non-familier qui loge au cœur même du familier, c'est l'inquiétante intimité et l'intime inquiétude de l'être, de chacun de nous, sur son identité, sur la certitude de soi. C'est le "primitif » accroupi au foyer du "civilisé», c'est la superstition narguant la raison de l'être éclairé, c'est la violence des pulsions de l'esthète, bref, notre part d'ombre à tous. En d'autres termes, c'est l'étranger que nous nous découvrons être pour nous-mêmes lorsque la conscience se trouve débordée par l'inattendu, confrontée à un danger inhabituel, face à des émotions fortes imprévues que la raison n'a pas eu la possibilité de filtrer ou devant des actes, les nôtres, qu'elle ne comprend pas. Poussée à l'échelle d'une civilisation, occidentale en l'occurrence, c'est l'incompréhension radicale qui surgit devant, par exemple, la juxtaposition: camps d'extermination nazis et musique de Bach (c'est à dessein que je ne choisis pas ici celle de Wagner). Deux extrêmes nés à très peu d'intervalle, finalement, au même lieu, d'une même culture. Mais est-ce bien la même? Oui et non. Ce même devient justement suspect devant un tel contraste. On ne s'y reconnaît plus. Certains officiers SS qui travaillaient dans les

2. Par exemple: «La pensée est l'acte de se joindre dans l'Autre avec soi-même», F. Hegel, Encyclopédie des sciences philosophiques (Paris, Vrin, 2004), 159.

3. S. Freud, «Das Unheimliche» (1919), Imago, Bd. 5, 297-324. 
camps adoraient jouer ou entendre jouer le Clavier bien tempéré et chérissaient leurs enfants. Voilà qui peut-être dérange plus encore, mentalement, que l'extermination elle-même (si seulement les Allemands étaient des barbares, quel soulagement!). On rejoint ici la banalité du mal évoquée par Hannah Arendt à propos d'Eichmann ${ }^{4}$.

La couche de la civilisation est d'une minceur et d'une fragilité inquiétantes. Nous ne sommes pas assez profondément civilisés pour admettre et affronter l'existence en nous du barbare, qui doit être à la fois refoulé et expulsé. Le barbare renvoie donc nécessairement à l'Autre du moment que cet Autre est par définition notre contraire. Mais il est aussi notre semblable. Nous voulons bien qu'il le soit dans ce qu'il contient à nos yeux de positif (ne serait-ce que potentiellement - à moins de lui dénier toute humanité, toute ressemblance humaine avec nous), mais il ne saurait nous ressembler dans ce que nous détestons. En quoi l'Autre est manifestement l'incarnation extérieure du mal. Il a du coup pour fonction occulte de porter pour nous, à notre place et à notre bénéfice, ce que nous ne supporterions pas de découvrir en nous. L'Autre barbare nous protège à notre insu de notre propre barbarie. Laquelle peut dès lors s'exercer impunément contre lui, du moment qu'elle n'est que réponse momentanée, atténuée, modérée, civilisée, à la sienne, laquelle est par principe essentielle, fondamentale, radicale. C'est ce qui se joue dans notre rapport à l'Islam et, plus spécifiquement, dans notre comportement face aux groupes radicaux qui s'en réclament pour lutter contre l'emprise occidentale.

\section{HISTOIRE}

Huntington, à partir d'une tradition orientaliste discutable et mal digérée, a vulgarisé pour nous la réponse dont nous éprouvions sourdement le besoin $^{5}$. Les civilisations sont ce qu'elles sont, essentiellement incompatibles. Et certaines le seraient en quelque sorte plus que d'autres, en ce qu'elles contreviennent au grand courant de l'histoire, comme le fait l'Islam. Les représentations de Huntington fonctionnent à merveille en ce qu'elles épousent parfaitement le fonctionnement défensif de la psyché. Ce qu'il raconte sur le choc des civilisations, comme chacun sait, ne tient pas historiquement ni logiquement, mais son argumentation parle à notre imaginaire. Elle n'est ni fausse ni vraie, elle est simplement opérationnelle dans ses effets morbides.

4. H. Arendt, Eichmann à Jérusalem. Rapport sur la banalité du mal (Paris, Gallimard, 1997) (1966).

5. Samuel P. Huntington, Le choc des civilisations (Paris, Éditions Odile Jacob, 2000). 
Avec la fin de la guerre froide et le démembrement de l'empire soviétique, l'Occident était sérieusement en danger de se retrouver seul face à lui-même, seul et sans alibi devant sa propre crise, devant ses propres monstres, devant le vide d'une pensée incapable de rêver autre chose que la poursuite d'une accumulation sans fin, bref, devant la croissance de son propre désert. Il fallait donc trouver à l'extérieur un diable de rechange, celui-là même d'ailleurs qu'on n'avait pas hésité à monter comme un contre-feu susceptible de stopper la «menace» soviétique. Celle-ci disparue, le vent a tourné et le contre-feu est devenu feu, les mouvements islamistes, suscités et encouragés pour faire échec à l'URSS, se sont retournés contre nous. Ce revirement n'est pas une surprise ni même l'effet d'une erreur de la part de nos stratèges mondiaux mais, de leur point de vue, une inavouable bénédiction. Les mouvements islamistes radicaux constituent le nouvel épouvantail vers lequel notre civilisation projette ses peurs pour tenter d'échapper à la nécessité de regarder ce qui pourrait réveiller ces dernières en elle. Nous ne sommes donc pas devant une simple manipulation de l'opinion par nos dirigeants, mais bien devant un phénomène profond qui donne prise à une redoutable exploitation politique. Reste à comprendre comment notre regard sur l'Islam peut être embrigadé dans une telle politique. Pourquoi l'Islam et non pas une autre civilisation?

L'histoire n'est pas aussi simple que celle à laquelle on se borne trop souvent à adhérer. Les affrontements qui se sont produits en Méditerranée postérieurement à l'avènement de l'Islam n'étaient pas nouveaux. Le bassin méditerranéen a toujours été, malgré la brève période de la pax romana, un lieu d'affrontements. Une certaine nostalgie de l'unité romaine, des siècles plus tard, a contribué à voir dans l'expansion de l'Islam le facteur d'une césure nouvelle et irrémédiable, inhérente à la nature même de cette religion. Cette vision de l'expansion musulmane (aux viI ${ }^{\mathrm{e}}$ et $\mathrm{vIII}^{\mathrm{e}}$ siècles de l'ère chrétienne) n'est nullement celle des peuples contemporains qui habitent alors l'Europe, ni même celle du Bas Moyen Âge chrétien. Elle n'est pas partagée non plus par les gens de la Renaissance. Elle est résolument moderne, et on pourrait même dire qu'elle date du siècle dernier $\left(\right.$ le $\left.\mathrm{xx}^{\mathrm{e}}\right)$.

Les croisades ne sont que superficiellement une guerre de religion et, dans les limites de cette restriction, ne revêtent ce caractère partiellement religieux que du point de vue des envahisseurs germano-latins. Les chrétiens de la Méditerranée orientale sont totalement étrangers à la mentalité qui consiste à guerroyer au nom du Christ. Ils seront d'ailleurs les princi- 
pales victimes de ces expéditions guerrières. Il est vrai qu'au $\mathrm{xI}^{\mathrm{e}}$ et $\mathrm{XII}^{\mathrm{e}}$ siècles, l'Islam et son expansion religieuse inquiètent l'Église. L'hostilité, plutôt tardive finalement, des autorités catholiques contre l'hérésie mahométane ne fait alors aucun doute. Mais sa virulence n'affecte guère les échanges ni les emprunts artistiques et scientifiques de l'Europe savante auprès des maîtres musulmans. Les mêmes clercs qui combattent le Coran se réclament ouvertement de la philosophie arabe. Au xvi ${ }^{\mathrm{e}}$ siècle encore, Bodin et d'autres penseurs de la politique désignent l'organisation de l'empire ottoman et la forme turque de la piété en exemple. Il faut attendre l'âge classique et la montée en force de la science expérimentale pour que commence à apparaître chez certains esprits d'Europe occidentale l'idée d'un Orient attardé dans son immobilisme, image qui ne se répandra largement qu'à partir de la seconde moitié du XviII ${ }^{\mathrm{e}}$ siècle. C'est avec les Lumières que l'autre civilisé (par contraste avec l'altérité sauvage) devient l'objet d'un regard condescendant et que l'Islam apparaît, notamment dans la philosophie hégélienne de l'histoire, comme une civilisation qui, malgré sa brillance, et parce que née en marge du christianisme, ne s'inscrit pas dans le grand courant de l'histoire universelle que ce dernier prolonge. L'Islam a laissé de beaux restes, mais il est mort en tant que force civilisatrice active, il a été charbon ardent mais l'histoire l'a réduit à l'état de scorie.

Aujourd'hui, la scorie s'est rallumée et brûle dangereusement. Le moribond resurgit du mausolée qu'on a dressé pour lui et vient troubler notre quiétude. Il se dresse contre la marche de l'histoire. Et il le fait avec nos propres armes. La technique moderne au service d'un combat réactionnaire, retardataire et obscurantiste. Les attaques (verbales et matérielles) de la contestation islamiste portent sur deux points sensibles: d'une part, elles remettent en question du même élan la portée de notre suprématie technique et l'universalité de nos valeurs; d'autre part, elles excitent les monstres assoupis de notre civilisation comme les banderilles la fureur du taureau. Si nous étions si sûrs de nos valeurs et de notre science (laquelle n'est d'ailleurs pas simplement "nôtre»), les forces dispersées qui disent nous combattre ne pourraient rien contre nous. Bien plus, ces forces ne se manifesteraient tout simplement pas. Elles n'auraient pas d'ennemi.

Le combat "obscurantiste» contre la démocratie, qu'il soit verbal ou sanglant, suscite l'inquiétude chez nous, car il rejoint la conscience étouffée de notre propre malaise. Nous savons que notre démocratie est inique, que les iniquités y sont même croissantes, nous savons que la 
politique mondiale de nos gouvernements est inepte, arrogante, prédatrice, cynique. Bien plus, nous savons que les disparités presque inimaginables qui accompagnent la croissance sans fin de l'accumulation matérielle et financière feront un jour éclater les coutures de nos propres sociétés. La chute des tours jumelles à New York, les bombes de Madrid, la tuerie en Ossétie du Nord, au-delà du mal immédiat qu'elles font, sonnent le rappel des horreurs passées et sont les signes avant-coureurs des désastres à venir. C'est parce que ces crimes sont le reflet de notre propre violence, de notre propre injustice qu'ils nous touchent si gravement, bien au-delà des morts et des mutilés que nous voyons sur nos écrans. C'est parce qu'ils savent quelque chose de cette mauvaise ou fausse conscience qui est la nôtre que les auteurs de ces actes terroristes peuvent agir comme ils le font. Et ils continueront d'agir ainsi aussi longtemps que nous refuserons de nous avouer la peur que nous avons de nous-mêmes. L'idée partout répandue d'un terrorisme (ou de multiples terrorismes) à réprimer urbi et orbi constitue le plus sûr moyen d'entretenir notre peur et le meilleur combustible des actes sanglants qu'on prétend vouloir empêcher.

Il ne s'agit pas de justifier les actes terroristes, ni même de leur trouver des circonstances atténuantes (du genre: «après, tout, ils ne font que se défendre» ou "ils ne font que nous renvoyer la monnaie de notre pièce»). Leurs commanditaires sont aussi barbares que nos dirigeants, et finalement tout ce petit monde, par l'intermédiaire de truands aguerris ou d'idéalistes chauffés à blanc, brasse le même argent, le même pétrole, les mêmes explosifs. Cette connivence n'a rien d'un complot, elle est dans l'ordre inique des choses, elle n'est possible que parce que tous, grands et petits potentats, élus ou non élus, s'entendent sur cet ordre, sans même avoir à se le dire. Ils sont notamment tous convaincus que les «gains» de la démocratie ne sont que faux-semblants. À la différence que les uns croient devoir sauver les apparences et les autres gagner une certaine popularité à les discréditer. Ce sont les deux faces de la même pièce. Il n'y a pas de grand complot, hélas, mais une série de médiocres complots, encouragés par la crise d'une civilisation qui ne reconnaît ni ses échecs ni son déclin et qui fuit devant l'angoisse inavouable qu'elle s'inspire.

Cette fuite paraît étrange, voire contredite par la profondeur et la virulence avec lesquelles la philosophie, l'art, la littérature, la science, en Occident, remettent périodiquement notre civilisation en question. On pourrait même dire sans exagérer que notre civilisation est sans doute celle qui, jusqu'à maintenant, a poussé le plus loin la critique de soi. Mais 
cette critique ne pénètre la conscience collective que sous la forme d'une inquiétude générale, inarticulée, imprécise. En d'autres termes, elle n’est pas suffisamment analysée, y compris, souvent, chez les minorités contestataires qui la formulent. Cette insuffisance analytique apparait particulièrement béante lorsque nous nous trouvons devant la critique qui provient de l'Autre. Non seulement du fait que cette critique prend parfois des formes plus violentes, mais surtout parce qu'elle vient censément de plus barbares que nous. Dans la mesure où l'Autre, nous l'avons dit, porte manifestement à nos yeux ce qui demeure inavouable en nous, sa critique en devient ipso facto irrecevable, invalide. Notre conscience ne peut recevoir de l'extérieur ce qu'elle refuse ou peine à voir à l'intérieur. Malgré le malaise qu'elle éprouve, malgré l'esprit critique dont elle est capable. Dès que l'Autre entre en jeu, la résistance se fait si forte que la conscience de notre capacité autocritique devient le bouclier le plus solide contre toute flèche externe. L'Autre n'a rien à nous dire que nous ne nous soyons déjà dit sur nous-mêmes. Il n'a rien à nous apprendre que nous ne sachions déjà. Mais dire de l'Autre qu'il n'a rien à nous apprendre, y compris sur lui-même, revient tout simplement à lui dénier son statut de sujet. C'est finalement sa parole revendicatrice, en dépit de toutes les protestations du contraire, que nous refusons d'entendre. Et ce refus est proportionnel dans sa force à la puissance matérielle de celui qui l'exprime. La puissance du puissant est à ses yeux le plus sûr signe de sa compréhension du monde, d'un monde qu'il croit pouvoir maîtriser, dont l'Autre n'a rien à lui enseigner, et où cet Autre ne peut révéler que la rage de sa faiblesse et de sa jalousie.

Si l'Islam est aujourd'hui cet Autre par excellence, c'est qu'il est le système de croyance qui ressemble le plus au nôtre. L'islam, comme le christianisme et la philosophie de la modernité qui en est issue, est une idéologie de vérité. L'homo islamicus et l'homo modernus affirment tous deux avec la même force: «la vérité existe, je l'ai rencontrée». Et on pourrait aller jusqu'à dire, en ce sens, qu'ils partagent une aire civilisatrice commune. Chacun revendique le même terrain, seules les armes diffèrent. Là où l'islam, comme le christianisme, s'appuie sur la révélation, la pensée moderne se prévaut de la raison. Cette différence n'est pas si radicale qu'elle parait. La religion musulmane ne dédaigne pas la raison: tout comme la théologie chrétienne, elle la subordonne à un ordre plus élevé qui lui échappe.

Fille du christianisme, la pensée moderne ne renonce pas à la croyance, mais la déplace sur un autre objet: elle croit en une raison en soi qui n'est 
que $s a$ raison, mais qu'elle ne peut s'empêcher d'ériger en absolu, en remplacement de la révélation qu'elle abandonne. La raison se substitue à la révélation, si bien que rien de ce qui existe en dehors d'elle ne compte plus vraiment. La science et la technique seraient notre seul horizon de pensée. Ce que démentent, au sein même de la civilisation qui se prévaut de cette raison, les arts, la littérature et cette part la plus vivante de la science qui vraiment interroge. Le moderne croit qu'il ne croit pas, sans cesser de croire. Le musulman et le chrétien savent qu'ils croient. Cette conscience de croyant se fanatise au contact d'une civilisation adverse, la nôtre, qui la nie. Cette négation est aveugle à ses propres fondements. Aveugle, à son tour, la réaction furieuse qu'elle déclenche.

La seule attitude sensée devant cette spirale de la violence consiste, avant toute chose, avant toute tentative de dialogue avec l'Autre, d'aborder ce qu'on nomme "terrorisme» comme un problème interne à notre propre civilisation. Le "terrorisme», ce terme générique, globalement abusif, dont nous habillons l'Autre est le nom que, sans le savoir, nous donnons à l'effroi que provoque en nous notre propre étrangeté. Ce qui nous terrorise le plus, finalement, c'est nous. 